\title{
Energy spectrum, density of states and optical transitions in strongly biased narrow-gap quantum wells
}

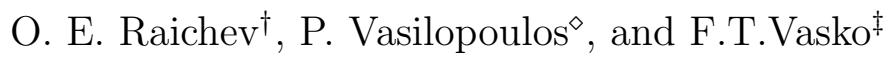 \\ 日Concordia University, Department of Physics, \\ 1455 de Maisonneuve Blvd. Ouest, Montréal, Québec, Canada, H3G 1 M8 \\ 母 Institute of Semiconductor Physics, National Academy of Sciences of Ukraine, \\ Pr. Nauki 45, Kiev-28, 252650, Ukraine
}

\begin{abstract}
We study theoretically the effect of an electric field on the electron states and far-infrared optical properties in narrow-gap lead salt quantum wells. The electron states are described by a two-band Hamiltonian. An application of a strong electric field across the well allows the control of the energy gap between the two-dimensional (2D) states in a wide range. A sufficiently strong electric field transforms the narrow-gap quantum well to a nearly gapless $2 \mathrm{D}$ system, whose electron energy spectrum is described by linear dispersion relations $\varepsilon_{\sigma}(k) \sim \pm\left(k-k_{\sigma}\right)$, where $k_{\sigma}$ are the field-dependent 2D momenta corresponding to the minimum energy gaps for the states with spin numbers $\sigma$. Due to the field-induced shift of the 2D subband extrema away from $k=0$ the density of states has inverse-square-root divergencies at the edges. This property may result in a considerable increase of the magnitude of the optical absorption and in the efficiency of the electrooptical effect.
\end{abstract}

PACS: 78.66.-w, 73.20.Dx, 78.20.Jq 


\section{INTRODUCTION}

The modification of electron spectra and of the optical absorption of semiconductor heterostructures by strong electric fields applied parallel to the growth direction (see band diagram in Fig.1) has important applications in infrared optical modulators and tunable photodetectors. Although the basic physics of these phenomena is related to the quantum confined Stark effect, their rigorous examination should take into account the complex band structure of the materials under investigation, especially in the case of interband optical transitions. The electric-field effect on electron spectra and optical absorption in quantum wells has been studied for a wide class of the materials: AlInAs/GaInAs ${ }^{1}, \mathrm{Si} / \mathrm{Ge}^{2}$, and narrow-gap CdTe/HgTe ${ }^{3}$ and InAs $/ \mathrm{AlGaSb}^{4}$ systems. The use of narrow-gap materials is especially important in connection with an increasing interest in the optics of mid- and far-infrared regions. For this reason, heterostructures based on narrow-gap lead salt alloys ${ }^{5}$ may become promising.

The electron spectrum of the lead salt alloys at the L-points of the Brillouin zone is described by an anisotropic two-band Dirac-like Hamiltonian and the energy gap strongly depends on the alloy composition. The most extensively investigated alloy, $\mathrm{Pb}_{1-x} \mathrm{Sn}_{x} \mathrm{Te}$, becomes a gapless material at $x \simeq 0.47$. Electronic properties including energy spectrum, optical transitions and electron transport of multilayer heterostructures based upon $\mathrm{Pb}_{1-x} \mathrm{Sn}_{x} \mathrm{Te}$ materials, have been studied in Refs. 6-8. Theoretical investigations of the electron energy spectrum of a single PbSnTe quantum well are summarized in Ref. 9, and interband optical transitions in such quantum wells have been studied in Ref. 10. The effect of weak electric fields on the energy spectrum and optical absorption has been studied in Ref. 11, where approximate analytical solutions of the eigenvalue problem have been found by perturbation theory. In that case, the Stark shifts of the levels are quadratic in the electric field and

comparable to the spin splitting energies due to field-induced asymmetry of the confining potential.

In this paper we study theoretically the influence of a strong electric field on the energy 
spectrum, density of states and optical absorption of quantum wells made from narrowgap lead salt alloys. Our investigation is motivated as follows. From the point of view of fundamental physics it is important to study the eigenvalue problem for the quantum well in the strong-field regime, when the drop of the electrostatic potential energy across the well becomes comparable to both the size-quantized energies and the energy gap of the quantum-well material. As we show below, the electron spectrum in such conditions has a pronounced spin splitting and an anticrossing of two-dimensional (2D) conduction- and valence-band states as they are shifted towards each other by the electric field. As a result, a nearly gapless $2 \mathrm{D}$ electron system is formed. The spectra of both spin-up and spin-down states become non-monotonic, the positions of the $2 \mathrm{D}$ subband extrema in the momentum space are moved by the electric field so that the density of states acquires inverse-squareroot divergencies near its edges. These properties are also important from the point of view of applied physics because an application of a strong electric field allows one to vary the energy gap between conduction and valence subbands in a wide range, sweeping the energy of fundamental optical transitions across the entire far-infrared region. Moreover, due to the described peculiarity of the density of states, the optical absorption is strongly enhanced in the frequency regions corresponding to the optical transitions of electrons near the subband extrema.

The paper is organized as follows. In Sec. II we present the basic formalism for the calculation of the spectrum, the wave functions, and the density of states in the narrow-gap quantum wells. In Sec. III we show the results of a numerical calculation of the electron spectrum and of the density of states associated with the ground-state conduction and valence subbands, discuss their properties in strong fields, and support our discussion by analytical considerations. In Sec. IV we calculate the infrared optical absorption coefficient and its field dependence (electrooptic effect) for different polarizations of the incident radiation and discuss the characteristic features of the absorption. In Sec. V we make concluding remarks. The Appendix contains general expressions for the wave functions of the two-band model in terms of standard special functions. 


\section{GENERAL FORMALISM}

Within the $\mathbf{k} \cdot \mathbf{p}$-formalism, the electron states near the L-points of the Brillouin zone in lead salt alloys are described by an anisotropic two-band Hamiltonian. Below we assume that the heterostructure containing a quantum well is grown along the (111) crystallographic direction and consider the states belonging to those two valleys whose large-effective-mass axis is (111). The states belonging to the other six valleys are characterized by a considerably smaller effective mass in the growth direction and, as a result, have higher size-quantization energies $^{12}$. Since we are interested in the ground valence- and conduction-band states, we remove these high-energy states from consideration. Thus, the columnar eigenstates $\Psi=(\varphi, \chi)$ in the region inside the quantum well $(-d / 2<z<d / 2)$ satisfy the matrix equation $(\hat{H}-\varepsilon) \Psi=0$ written explicitly as (in all equations or formulas we put $e=\hbar=1$ )

$$
\left(\begin{array}{cc}
\frac{\Delta}{2}+F z-\varepsilon & P_{\|} \frac{\partial}{\partial z}+P_{\perp} \sigma k \\
-P_{\|} \frac{\partial}{\partial z}+P_{\perp} \sigma k & -\frac{\Delta}{2}+F z-\varepsilon
\end{array}\right)\left(\begin{array}{l}
\varphi(z) \\
\chi(z)
\end{array}\right)=0 .
$$

Here $\Delta$ is the energy gap of the material inside the quantum well, $F$ is the magnitude of the applied electric field, $\sigma= \pm 1$ is the spin quantum number, $k$ is the absolute value of $2 \mathrm{D}$ momentum (here and below the $2 \mathrm{D}$ momentum is counted from the L-point), and $P_{\|}$and $P_{\perp}$ are the matrix elements of velocity operators in the directions parallel and perpendicular to (111) axis. The energy $\varepsilon$ is counted from the middle point of the band gap. A general solution of Eq. (1) can be expressed in terms of the special functions, see Appendix for details.

Equation (1) represents a set of two first-order differential equations. The most general boundary conditions for such a set have the form

$$
\chi(-d / 2)=\alpha_{l} \varphi(-d / 2), \quad \chi(d / 2)=\alpha_{r} \varphi(d / 2)
$$

where the coeffecients $\alpha_{l}$ and $\alpha_{r}$ characterize the left and right boundaries. If the barriers of the quantum-well heterostructure are made from a material which is also described by a two-band Hamiltonian, cf. Fig. 1, an explicit form of these coefficients can be derived 
from the requirement of continuity of both $\varphi(z)$ and $\chi(z)$ across the boundaries. A simple calculation, done under the assumption that $P_{\|}$and $P_{\perp}$ do not change across the interfaces, gives

$$
\begin{gathered}
\alpha_{r}=\sqrt{u_{c}^{+} / u_{v}^{-}+\left(P_{\perp} k / u_{v}^{-}\right)^{2}}+P_{\perp} \sigma k / u_{v}^{-} \\
\alpha_{l}=-\sqrt{u_{c}^{-} / u_{v}^{+}+\left(P_{\perp} k / u_{v}^{+}\right)^{2}}+P_{\perp} \sigma k / u_{v}^{+}
\end{gathered}
$$

where $u_{c}^{ \pm}=U_{c}+\Delta / 2 \pm F d / 2-\varepsilon$ and $u_{v}^{ \pm}=U_{v}+\Delta / 2 \pm F d / 2+\varepsilon$, and $U_{c}$ and $U_{v}$ are the conduction- and valence-band offsets defined in Fig. 1. If the barriers are high enough to neglect $\Delta, F d, \varepsilon$ and $P_{\perp} k$ in comparison to $U_{c}$ and $U_{v}$, Eqs. (3) and (4) take a simple form ${ }^{11}$ $\alpha_{r, l}= \pm \sqrt{U_{c} / U_{v}}$.

If $F=0, \mathrm{Eq}$. (1) with boundary conditions (2) is solved exactly, see also Ref. 9. The wave functions take the form $\varphi=c_{1} e^{i q z}+c_{2} e^{-i q z}$ and $\chi=c_{3} e^{i q z}+c_{4} e^{-i q z}$, where $q=$ $\sqrt{\varepsilon^{2}-\Delta^{2} / 4-P_{\perp}^{2} k^{2}} / P_{\|}$. An application of the boundary conditions leads to the dispersion relation

$$
\tan (q d)=\frac{\left(\alpha_{r}-\alpha_{l}\right) P_{\|} q}{\left(1+\alpha_{l} \alpha_{r}\right) \varepsilon-\left(1-\alpha_{l} \alpha_{r}\right) \Delta / 2-\left(\alpha_{l}+\alpha_{r}\right) P_{\perp} \sigma k}
$$

describing a set of nonparabolic 2D subbands. Even if the quantum well is formed by a gapless semiconductor $(\Delta=0)$, the size quantization effect always produces a finite energy gap between the valence- and conduction-band states.

Equation (1) has an important symmetry property. It is invariant with respect to the transformations $\varepsilon \rightarrow-\varepsilon, z \rightarrow-z, \varphi(z) \rightarrow \chi(-z)$, and $\chi(z) \rightarrow-\varphi(-z)$. This means that the energy spectrum of electrons is symmetric with respect to $\varepsilon=0$ if the boundary conditions allow the above-described transformation of the wave function. Therefore, the symmetry of the spectrum exists for

$$
\alpha_{l}(\varepsilon) \alpha_{r}(-\varepsilon)=-1
$$

If the boundary coefficients are given by Eqs. (3) and (4), Eq. (5) is equivalent to $U_{c}=U_{v}$. The symmetry of the spectra in such conditions is evident from a qualitative point of view. 
Another important property is the removal of the spin degeneracy by the electric field $F$. If $\alpha_{l}(\varepsilon)=-\alpha_{r}(\varepsilon)$, i.e., if the left and right boundary potentials are equal, and $F=0$, the spectra of the states with different spin numbers are the same. Applying an electric field introduces an asymmetric potential and renders the spectra of $\sigma=1$ and $\sigma=-1$ states different. This removal of spin degeneracy is a consequence of the spin-orbit interaction and it is typical for asymmetric heterostructures ${ }^{13}$.

If the electron energy spectrum $\varepsilon_{n \sigma}(k)$ of the Hamiltonian of Eq. (1) is known, one can find the density of electron states $\rho(\varepsilon)$ according to

$$
\rho(\varepsilon)=\sum_{n \sigma} \int_{0}^{\infty} \frac{k d k}{2 \pi} \delta\left(\varepsilon-\varepsilon_{n \sigma}(k)\right)
$$

Using the well-known identity $\partial \varepsilon_{n \sigma}(k) / \partial k=\left\langle\Psi_{n \sigma k}|\partial \hat{H} / \partial k| \Psi_{n \sigma k}\right\rangle$, and taking advantage of $\partial \hat{H} / \partial k=\sigma P_{\perp} \hat{\sigma}_{x}$, where $\hat{\sigma}_{x}$ is a Pauli matrix, we can rewrite Eq. (6) as

$$
\rho(\varepsilon)=\sum_{n \sigma i} \frac{k_{i}}{4 \pi P_{\perp}}\left|\int d z \varphi_{n \sigma k_{i}}(z) \chi_{n \sigma k_{i}}(z)\right|^{-1},
$$

where $k_{i}$ are the roots of the equation $\varepsilon=\varepsilon_{n \sigma}(k)$.

\section{ENERGY SPECTRUM AND DENSITY OF STATES}

As shown in the Appendix the general solution of Eq. (1) can be expressed in terms of the parabolic cylinder functions. However, the analysis of the results becomes more difficult than a direct numerical solution of Eqs. (1) and (2). Below we describe the results of such a direct solution based on the application of the 4th order Runge-Kutta procedure to Eq. (1) and subsequent determination of the energies which satisfy Eq. (2). In all calculations we use interband velocities taken from Ref. $6: \hbar P_{\|}=0.14 \mathrm{eV} \mathrm{nm}$ and $\hbar P_{\perp}=0.47 \mathrm{eV} \mathrm{nm}$. Figures 2 and 3 show the evolution of the spectrum and of the density of states for two ground-state $2 \mathrm{D}$ subbands with the increase of the electric field $F$ at $d=12 \mathrm{~nm}, \alpha_{l}=-1$, and $\alpha_{r}=1$. The chosen boundary conditions may correspond to a symmetric heterostructure with infinitely large but equal conduction- and valence-band offsets. For the sake of convenience $\varepsilon_{n \sigma}(k)$ is 
plotted as a function of $\sigma k$ so that the left sides of Figs. 2 (a) and 3 (a) describe the states with $\sigma=-1$, while the right sides describe those with $\sigma=1$. Regardless of the gap widths, the electric field introduces similar qualitative changes to the spectrum.

First we see Stark shifts and spin splitting. The 2D subband extrema for the $\sigma=-1$ states are shifted away from $k=0$. Further, this shift becomes more pronounced and it is accompanied with a substantial decrease of the gap between the $2 \mathrm{D}$ subbands. When the field is stronger than $2 \times 10^{5} \mathrm{~V} / \mathrm{cm}$, the gap becomes very small, typically $1-2 \mathrm{meV}$, and the subband dispersion in the vicinity of the gap becomes linear. The position of the energy extrema in momentum space is given by a characteristic momentum $k_{-}$, which increases almost linearly with the increase of the field. This shift is accompanied with an exponentially fast decrease of the gap. At higher fields, this kind of behavior repeats itself for the $\sigma=1$ states. Even at weak fields there exists a shift of the subband extrema away from $k=0$. It produces an inverse-square-root divergence of the density of states at the edge. At stronger fields, when the electron dispersion for both spin-up and spin-down states becomes non-monotonic, similar divergences also appear near the extrema points above the edges so that the density of states becomes rather complex. Figures 2 (b) and 3 (b) also show bump-like features of the density of states for the energies corresponding to zero momentum because the states with different spins give different contributions to the density of states above and below these energies. A similar calculation for narrower wells demonstrates all the features explained above. However, they appear at higher fields than for wider wells.

For an explanation of the small gaps and linear energy spectra near the extrema points in strong electric fields, it is instructive to take a look at the wave functions $(\varphi, \chi)$ for conduction and valence subbands at $k=k_{-}$, see Fig. 4. The electron density is high near the interfaces and low near the center of the well. In other words, the strong electric field makes the electron states at $k \sim k_{-}$localized in narrow regions near the interfaces. An analysis of Eq. (1) leads to the same conclusion. For more clarity we suppose that the condition (5) is fulfilled so it is sufficient for our purposes to consider small-energy solutions, $\varepsilon \ll F d$. Let us consider the region near the left boundary and introduce $z=-d / 2+d y$, 
where $y \ll 1$. Then, expressing $\chi$ through $\varphi$ we reduce Eq. (1) to

$$
-\frac{\partial^{2}}{\partial y^{2}} \varphi(y)+\left[\left(\frac{F d^{2}}{P_{\|}}\right)^{2} y-\lambda\right] \varphi(y)=0
$$

where

$$
\lambda=R(\sigma k, \varepsilon) \equiv\left(\frac{F d^{2}}{2 P_{\|}}\right)^{2}-\left(\frac{P_{\perp} d k}{P_{\|}}\right)^{2}-\left(\frac{\Delta d}{2 P_{\|}}\right)^{2}-2 \frac{P_{\perp} d \sigma k}{P_{\|}(1+\Delta / F d)}+\frac{F d^{3}}{P_{\|}^{2}} \varepsilon
$$

while the boundary condition for $\varphi(y)$ at $y=0(z=-d / 2)$ becomes

$$
\left.\left[\frac{\partial}{\partial y}+\alpha_{l} \frac{F d^{2}}{2 P_{\|}}\left(1+\frac{\Delta}{F d}\right)-\frac{P_{\perp} d \sigma k}{P_{\|}}\right] \varphi(y)\right|_{y=0}=0 .
$$

Equation (8) is valid for $y \ll 1$. To make a characteristic spatial scale of its solutions small in comparison to unity, we should also impose the requirement $\left(F d^{2} / P_{\|}\right)^{2 / 3} \gg 1$, which is fulfilled for $d \sim 10 \mathrm{~nm}$ and $F>10^{5} \mathrm{eV} / \mathrm{cm}$. We also assume that $F d>\Delta$.

Equation (8) with the boundary condition (10) describes a one-dimensional quantummechanical problem for the spectrum and eigenfunctions of a particle localized in a triangular potential well formed by a barrier at $y=0$ and uniform field at $y>0$. It has discrete solutions $\lambda=\lambda_{n \sigma}(k)$, from which we take the ground-state solutions $\lambda_{0 \sigma}(k)$ corresponding to the nodeless wave functions $\varphi_{0 \sigma k}(y)$. If we solve the equation $\lambda_{0 \sigma}(k)=R(\sigma k, \varepsilon)$ for small energies, we obtain a linear spectrum $\varepsilon_{\sigma}(k)=A_{\sigma}\left(k-k_{\sigma}\right)$, where the characteristic velocities $A_{\sigma}$ are of the order of $P_{\perp}$ and the characteristic momenta $k_{\sigma}$, corresponding to $\varepsilon_{\sigma}(k)=0$, are of the order of $F d / P_{\perp}$. A similar consideration can be done for the right boundary by introducing $z=d / 2-d y$. Due to the symmetry of the problem, the equations for the right boundary are simply obtained from Eqs. (8)-(10) by replacing $\varphi$ by $\chi$ and $\varepsilon$ by $-\varepsilon$ only. The second replacement is significant, because it leads to $\varepsilon_{\sigma}(k)=-A_{\sigma}\left(k-k_{\sigma}\right)$. Thus, for each spin number we have two linear branches intersecting each other at $\varepsilon=0$ and $k=k_{\sigma}$. This description would be completely correct if there were no overlap between the states localized near each boundary. Really, such an overlap always exists, and it produces an anticrossing near $\varepsilon=0$. As a result, a small but finite energy gap appears. This gap becomes progressively smaller with the increase of the electric field. From a qualitative point 
of view, the described effect can be explained as a result of the anticrossing of the conduction and valence subbands as they are shifted towards each other by the electric field.

Figure 2 demonstrates another remarkable feature, namely that the energy spectra of a zero-gap quantum well at $k=0$ are independent of the electric field, see Fig. 2 (a). This property can be rigorously proved because Eq. (1) is exactly solvable at $\Delta=0$ and $k=0$, regardless of the strength of the electric field. In these conditions Eq. (1) is diagonalized by the unitary transformation $\varphi=(\tilde{\varphi}+i \tilde{\chi}) / \sqrt{2}$ and $\chi=(\tilde{\chi}+i \tilde{\varphi}) / \sqrt{2}$; we then obtain the equations

$$
\begin{gathered}
{\left[i P_{\|} \frac{\partial}{\partial z}+F z-\varepsilon\right] \tilde{\varphi}=0,} \\
{\left[-i P_{\|} \frac{\partial}{\partial z}+F z-\varepsilon\right] \tilde{\chi}=0,}
\end{gathered}
$$

whose solutions are straightforward. We have

$$
\varphi=C_{1} \cos \psi_{z}+C_{2} \sin \psi_{z}, \chi=C_{2} \cos \psi_{z}-C_{1} \sin \psi_{z},
$$

where $\psi_{z}=\left(F z^{2} / 2-\varepsilon z\right) / P_{\|}$. Applying the boundary conditions gives the dispersion relation

$$
\tan \left(\frac{\varepsilon d}{P_{\|}}\right)=\frac{\alpha_{r}-\alpha_{l}}{1+\alpha_{l} \alpha_{r}}
$$

Thus, the quantization energies described by Eq. (14) are independent of the electric field if the boundary coefficients are field-independent. If $\alpha_{l}$ and $\alpha_{r}$ are energy-independent, Eq. (14) describes a ladder of equally spaced levels. Further, if Eq. (5) is fulfilled, the spectrum at $k=0$ is always field-independent. It is given by $\varepsilon=(n+1 / 2)\left(\pi P_{\|} / d\right)$, where $n$ is an integer.

The electric fields required for the anticrossing are rather strong and the change of the electrostatic potentials across the well are comparable to the band offsets $U_{c}$ and $U_{v}$ of PbSnTe heterostructure. To demonstrate that the effects still exist for realistic band offsets, we have done a calculation of the spectrum and density of states for a quantum well made of a gapless $\mathrm{Pb}_{1-x} \mathrm{Sn}_{x} \mathrm{Te}$ alloy layer enclosed by $\mathrm{SnTe}$ layers. For such a system, we used the 
parameters $U_{c}=0.25 \mathrm{eV}$ and $U_{v}=0.08 \mathrm{eV}$, taken from Ref. 6, and employed the boundary conditions (3) and (4). The calculated energy spectrum for a $12 \mathrm{~nm}$ wide quantum well is shown in Fig. 5. Since Eq. (5) is no longer valid in this case, both the spectrum and the density of states are not symmetric with respect to $\varepsilon=0$. For this reason, we plot the density of states for both conduction and valence subbands. Due to the dependence of the boundary conditions on the energy, momentum, and electric field, discrete solutions do not always exist. For example, at $F=1.5 \times 10^{5} \mathrm{~V} / \mathrm{cm}$ the region of their existence becomes rather narrow. Nevertheless, the salient features shown in Figs. 2 and 3 are reproduced in Fig. 5.

The divergences of the density of states arising near the extrema of the electron energy spectra can be studied by different means, including measurements of the thermodynamic quantities such as specific heat and capacitance, optical transmission and absorption measurements, and by transport measurements. Considering possible applications of the biased narrow-gap quantum wells to optical detectors and modulators, in the next section we evaluate the spectral and field dependence of the far-infrared optical absorption coefficient and show how the special features of the spectrum manifest themselves in the optical transitions.

\section{FAR-INFRARED OPTICAL TRANSITIONS}

In the dipole approximation, the optical absorption coefficient $\xi$ is given by ${ }^{13}$

$$
\xi=\frac{2 \pi e^{2}}{\omega \hbar^{2} c \sqrt{\kappa}} \sum_{n n^{\prime}, \sigma \sigma^{\prime}} \int_{0}^{\infty} k d k\left|M_{n \sigma, n^{\prime} \sigma^{\prime}}(k)\right|^{2} \delta\left(\varepsilon_{n \sigma}(k)-\varepsilon_{n^{\prime} \sigma^{\prime}}(k)+\hbar \omega\right),
$$

where $\omega$ is the frequency of the infrared radiation, $\kappa$ the dielectric permittivity, and $M_{n \sigma, n^{\prime} \sigma^{\prime}}(k)$ the matrix elements for transitions between the occupied subband states of the valence band and the empty subband states of the conduction band ( $n$ and $n^{\prime}$ are the subband numbers):

$$
\begin{aligned}
\left|M_{n \sigma, n^{\prime} \sigma^{\prime}}(k)\right|^{2}= & \delta_{\sigma \sigma^{\prime}}\left[(\mathbf{e} \cdot \mathbf{z})^{2} P_{\|}^{2} \Phi_{n \sigma, n^{\prime} \sigma^{\prime}}^{(-) 2}(k)+(1 / 2)[\mathbf{e} \times \mathbf{z}]^{2} P_{\perp}^{2} \Phi_{n \sigma, n^{\prime} \sigma^{\prime}}^{(+) 2}(k)\right] \\
& +(1 / 2)\left(1-\delta_{\sigma \sigma^{\prime}}\right)[\mathbf{e} \times \mathbf{z}]^{2} P_{\perp}^{2} \Phi_{n \sigma, n^{\prime} \sigma^{\prime}}^{(+) 2}(k) .
\end{aligned}
$$


Here $\mathbf{e}$ is the unit vector of polarization of the infrared radiation, $\mathbf{z}$ is the unit vector in the direction perpendicular to the 2D plane, and

$$
\Phi_{n \sigma, n^{\prime} \sigma^{\prime}}^{( \pm)}(k)=\int_{-d / 2}^{d / 2} d z\left[\varphi_{n \sigma k}(z) \chi_{n^{\prime} \sigma^{\prime} k}(z) \pm \chi_{n \sigma k}(z) \varphi_{n^{\prime} \sigma^{\prime} k}(z)\right]
$$

are the overlap integrals. By taking the limits from $-d / 2$ to $d / 2$, we neglect the barrier penetration of the wave functions, i.e., we assume that the main part of the electron density remains inside the well. The wave functions, therefore, should be normalized according to

$$
\int_{-d / 2}^{d / 2} d z\left[\varphi_{n \sigma k}^{2}(z)+\chi_{n \sigma k}^{2}(z)\right]=1
$$

Below we express the polarization-dependent absorption coeffecient as $\xi=\xi_{\perp}[\mathbf{e} \times \mathbf{z}]^{2}+$ $\xi_{\|}(\mathbf{e} \cdot \mathbf{z})$ and calculate $\xi_{\perp}$ and $\xi_{\|}$. In the case of normally incident radiation one has $\xi=\xi_{\perp}$, regardless of the polarization. Only two $2 \mathrm{D}$ levels, $n^{\prime}=c$ and $n=v$, corresponding to the ground conduction- and valence-band states, are involved in the calculation. Taking into account that the energy levels of realistic physical systems are always broadened by disorder, we also introduce a finite broadening by replacing the $\delta$-function in Eq. (15) by a Gaussian with a halfwidth of $1 \mathrm{meV}$. In this way we also avoid non-physical divergences of the optical absorption coefficient.

Figure 6 shows the frequency dependence of the absorption coefficients $\xi_{\perp}$ and $\xi_{\|}$for the quantum well described in Fig. 5. Due to the small ratio of $\left(P_{\|} / P_{\perp}\right)^{2}$, it appears that $\xi_{\|}$ is approximately ten times smaller than $\xi_{\perp}$. In zero field both $\xi_{\perp}$ and $\xi_{\|}$show a step-like behavior at the fundamental absorption edge ${ }^{10}$, which is typical for $2 \mathrm{D}$ systems. In non-zero field, when the densities of states for both valence and conduction subbands have inversesquare-root divergences near the subband extrema, the absorption shows sharp maxima near the threshold. This kind of behavior is typical for 1D systems. Even after the introduction of a $1 \mathrm{meV}$ broadening, the absorption near the edge still remains considerably larger than the zero-field absorption. It is important to note a qualitative difference between the frequency dependences of $\xi_{\perp}$ and $\xi_{\|}$caused by the fact that spin-flip transitions contribute to the first one but not to the second one. That is why $\xi_{\perp}$ shows jumps at the frequencies corresponding 
to transitions with $k=0$, while $\xi_{\|}$, roughly following the density of states, shows bump-like features at these frequencies.

Although the calculation according to Eq. (15) is done under the assumption that the Fermi level remains in the gap, it is not difficult to extend the formalism to the cases when the Fermi level remains inside the valence or conduction band. A result of the calculation of $\xi_{\perp}$ and $\xi_{\|}$at $F=10^{5} \mathrm{~V} / \mathrm{cm}$ for a $n$-type structure with $n=10^{11} \mathrm{~cm}^{-2}$ is also shown in Fig. 6. Due to the Pauli exclusion principle the maximum of the absorption at the threshold is suppressed. Moreover, due to a considerable asymmetry of the spectra with respect to their extrema points, cf. Fig 5. (a), there are actually two thresholds for direct optical transitions in a $n$-type quantum well. The first one, corresponding approximately to $\hbar \omega=11 \mathrm{meV}$, signals a transition with $k$ above the extremum point, while the second one, corresponding approximately to $\hbar \omega=17 \mathrm{meV}$, signals a transition with $k$ below the extremum point. For this reason $\xi_{\|}$has two steps. On the other hand, the second step is not visible in the frequency dependence of $\xi_{\perp}$. This is not surprising because the overlap integrals (17) for $\xi_{\|}$ and $\xi_{\perp}$ are different and it appears that transitions with $k$ below the extremum point have a minor contribution to $\xi_{\perp}$ near the threshold.

Figure 7 shows the field dependence of the absorption of the same quantum well at $\hbar \omega=12 \mathrm{meV}$. The absorption near the edge is very sensitive to the electric field. This fact is important in connection with possible applications of the biased narrow-gap quantum wells to optical modulators. With the importance and high sensitivity of differential spectroscopic measurements in mind, we also plot the derivatives $d \xi_{\perp} / d F$ and $d \xi_{\|} / d F$ in this figure.

We emphasize that the optical absorption coefficients calculated above correspond to a single valley. Since in (111) grown lead salt quantum wells there are two equivalent valleys described by Eq. (1), both $\xi_{\perp}$ and $\xi_{\|}$should be multiplied by a factor of 2 . 


\section{CONCLUSIONS}

We investigated the effect of a strong electric field on the energy spectrum and the infrared optical properties of narrow-gap quantum wells described by a two-band Dirac-like Hamiltonian. The corresponding eigenvalue problem was solved numerically. Apart from an examination of an electric-field control of the energy gap between the 2D states due to the Stark effect, we established that a strong electric field transforms any narrow-gap quantum well to a nearly gapless $2 \mathrm{D}$ system with an electron energy spectrum near the subband extrema described by linear dispersion laws. The physical reason for this behavior is described in terms of an anticrossing of the conduction- and valence-subband spectra and of the localization of the electron states near the well boundaries. In general, the energy spectrum is dramatically modified by the electric field, and the density of states, in addition to the anticipated inverse-square-root divergency at the edges, has similar divergences above the edges. The behavior of the density of states manifests itself in the interband optical absorption.

In connection with the interest in far-infrared optics, we emphasize the most important properties of the lead salt quantum wells caused by an application of a strong electric field: (i) a possibility to control the gap in a wide range including the far-infrared region, (ii) an enchancement of the optical absorption near the fundamental edge, and (iii) a high sensitivity of the absorption near the edge to the electric field. Due to the Pauli exclusion principle, the enchancement of the optical absorption would be suppressed in $n$ and $p$-type quantum wells, where the Fermi level remains inside the conduction or valence subbands. The position of the Fermi level is not determined only by the properties of the quantum well itself. Other factors, such as the geometry of the entire heterostructure and the remote doping profiles, are essential as well. Therefore, by proper choice of parameters, one can achieve the favorable conditions when the Fermi level is in the gap. However, the question of a possible electric-field induced shift of the Fermi level outside the gap still remains open.

Below we comment briefly on the approximations made in this paper. In the description 
of the eigenvalue problem for narrow-gap lead salts we restricted ourselves to the simple two-band Hamiltonian, neglecting higher-band contributions, which in principle could be accounted for with a set of Dimmock parameters ${ }^{5}$. We neglected strain effects, which introduce additional contributions ${ }^{6}$ to the Hamiltonian. We did not account for the difference between the velocities $P_{\|}$and $P_{\perp}$ in the wells and barriers. Such a difference would modify the boundary conditions, but if it is sufficiently small it can be neglected. We considered homogeneous electric fields only and neglected screening effects which may appear due to a redistribution of the electron density in the wells. However, due to the high static dielectric permittivity of the PbSnTe alloys, these effects should be considerably less important than in GaAs-based structures. Due to this high dielectric permittivity we were also able to neglect the Coulomb effects which otherwise would soften the sharp maxima of the (pair-) density of states. Further, we considered (111)-grown heterostructures only. This choice is justified because the lead salt heterostructures are typically grown on (111) cleaved $\mathrm{BaF}_{2}$ substrates $^{14}$. Finally, we neglected the broadening of the subband levels due to escape of electrons from the wells by field-assisted tunneling. Because of the small band offsets in $\mathrm{PbTe} / \mathrm{SnTe}$ systems, this effect is important and it essentially restricts the range of the electric fields which can be applied to the heterostructure. Nevertheless, the use of PbSnTe quantum wells, directly grown on $\mathrm{BaF}_{2}$ or $\mathrm{GaAs}^{15}$ substrates, would help to extend this range to higher fields.

In conclusion, we have described profound modifications of the electron energy spectra and density of states due to the anticrossing of 2D subbands under strong electric fields. The results were obtained by a direct numerical solution of Eq. (1) and, to our knowledge have not been published before although the analytical solution of Eq. (1), given in the Appendix, is known. Similar modifications are possible not only for lead salt quantum wells but also for systems based on other narrow-gap materials, for example, CdTe/HgTe and InAs/GaSb heterostructures. Apart from their influence on the far-infrared optical properties studied in this paper, the modifications of the spectra must have a substantial effect on transport and equilibrium properties of the narrow-gap low-dimensional electron systems. 


\section{ACKNOWLEDGMENTS}

This work was supported by le Ministére de l' Education du Québec and by the Canadian NSERC Grant No. OGP0121756.

\section{APPENDIX}

The general solution of Eq. (1) is expressed through the parabolic cylinder functions ${ }^{16}$ $D_{j}(x)$. With the definitions $\varphi=(\tilde{\varphi}+i \tilde{\chi}) / \sqrt{2}$ and $\chi=(\tilde{\chi}+i \tilde{\varphi}) / \sqrt{2}$ we have

$$
\begin{gathered}
\tilde{\varphi}=C_{1} D_{i \beta-1}(\sqrt{i} s)+C_{2} D_{i \beta-1}(-\sqrt{i} s) \\
\tilde{\chi}=(-\sqrt{i} \mu / \beta)\left[C_{1} D_{i \beta}(\sqrt{i} s)-C_{2} D_{i \beta}(-\sqrt{i} s)\right]
\end{gathered}
$$

where $s=\sqrt{2 F / P_{\|}}(z-\varepsilon / F), \sqrt{i}=(1+i) / \sqrt{2}, \beta=\left(\Delta^{2} / 4+P_{\perp}^{2} k^{2}\right) / 2 P_{\|} F$, and $\mu=$ $\left(\sqrt{\Delta^{2} / 4+P_{\perp}^{2} k^{2}}\right) /\left(-\Delta / 2+i P_{\perp} \sigma k\right)$. An application of the boundary conditions (2) to Eqs. (A1) and (A2) allows to get an implicit equation describing the electron spectrum. However, its analysis is more difficult than the direct numerical solution of Eqs. (1) and (2) which we followed in this paper. 


\section{REFERENCES}

$\dagger \quad$ E-mail: raichev@zinovi@lab2.kiev.ua

$\diamond \quad$ E-mail: takis@boltzmann.concordia.ca

[1] Y. Huang, J. Wang, and C. Lien, J. Appl. Phys. 77, 11 (1995).

[2] S. M. Cho and H. H. Lee, J. Appl. Phys. 73, 1918 (1993).

[3] Z. Wang and J. K. Furdyna, J. Appl. Phys. 64, 5248 (1988).

[4] S. Hong, J. P. Loehr, J. E. Oh, P. K. Bhattachaya, and J. Singh, Appl. Phys. Lett. 55, 888 (1989).

[5] G. Nimtz and B. Schlicht. Narrow-Gap Lead Salts, in Narrow-Gap Semiconductors. Schpringer-Verlag, 1983.

[6] M. Kriechbaum, K. E. Ambrosch, E. J. Fanter, H. Clemens, and G.Bauer Phys. Rev. B 30, 3394 (1984).

[7] M. Kriechbaum, P. Kocevar, H. Pasher, and G. Bauer, IEEE J. Quantum Electronics, 24, 1727 (1988).

[8] F. F. Sizov, V. V. Tetyorkin, and J. V. Gumenjuk-Sichevskaya, Superlatt. Microstruct. 9, 483 (1991).

[9] V. K. Dugaev and P. P. Petrov. Fiz. Tekh. Poluprov. 23, 488 (1989) [Semiconductors, 23, 305 (1989)]; Phys. Stat. Sol. (b) 184, 347 (1994).

[10] W. Okulski and M. Zaluzny, Thin Solid Films, 204, 239 (1991).

[11] F. T. Vasko and G. I. Steblovsky, Ukrainian Physical Journal 34, 576 (1989).

[12] In theoretical papers the electron states in quantum wells of narrow-gap lead salts are often described by an isotropic Dirac Hamiltonian. In that case, the states in all eight valleys are equivalent regardless of the growth direction. However, the isotropic model 
is not satisfactory for an application to both PbSnTe and PbSnSe materials.

[13] F. T. Vasko and A. Kuznetsov, Electronic States and Optical Transitions in Semiconductor Heterostructures. Springer, 1998.

[14] H. Kinoshita and H. Fujiyasu, J. Appl. Phys. 51, 5845 (1981).

[15] K. T. Pollard, A. Erbil, and R. Sudharsanan, J. Appl. Phys. 71, 6136 (1992).

[16] I. S. Gragshteyn and I. M. Ryzhik, Tables of Integrals, Series, and Products. (Academic, New York, 1980). 


\section{FIGURES}

FIG. 1. Schematic band diagram of a biased narrow-gap quantum well. The thick broken arrow shows an optical transition between Stark-shifted 2D subbands.

FIG. 2. Electron spectrum (a) and density of states per one valley (b) for a narrow-gap quantum well with boundary conditions $\alpha_{r}=-\alpha_{l}=1$, at $d=12 \mathrm{~nm}$ and $\Delta=0$. Each graph is marked with a value of the electric field $F$ in units of $10^{5} \mathrm{~V} / \mathrm{cm}$.

FIG. 3. The same as in Fig.2 at $\Delta=0.05 \mathrm{eV}$.

FIG. 4. Normalized wave functions $(\varphi, \chi)_{v, c}$ of ground valence- and conduction-band states for a biased narrow-gap quantum well at $\Delta=0, d=12 \mathrm{~nm}, F=2 \times 10^{5} \mathrm{~V} / \mathrm{cm}, \sigma=-1$ and $k \simeq$ $0.175 \mathrm{~nm}^{-1}$.

FIG. 5. Electron spectrum (a) and density of states per one valley (b) of a $12 \mathrm{~nm}$ wide narrow-gap quantum well calculated with realistic boundary conditions corresponding to SnTe/gapless PbSnTe interfaces. Each graph is marked with a value of $F$ in units of $10^{5} \mathrm{eV} / \mathrm{cm}$.

FIG. 6. Frequency dependence of the absorption coeffecients $\xi_{\perp}$ (solid curve) and $\xi_{\|}$(magnified tenfold, dashed curve) for the quantum well described in Fig. 5. Each graph is marked with a value of $F$ in units of $10^{5} \mathrm{~V} / \mathrm{cm}$. A $1 \mathrm{meV}$ broadening has been assumed. The thin lines illustrate the Pauli exclusion effect on the absorption for a $n$-type structure with $n=10^{11} \mathrm{~cm}^{-2}$ at $F=10^{5}$ $\mathrm{V} / \mathrm{cm}$.

FIG. 7. Electric-field dependence of the far-infrared absorption coefficients $\xi_{\perp}$ (solid curve) and $\xi_{\|}$(magnified tenfold, dashed curve) for the quantum well described in Fig. 5 at $\hbar \omega=12 \mathrm{meV}$. The first derivatives of these functions (scaled and shifted for clarity) are shown by thin lines. 


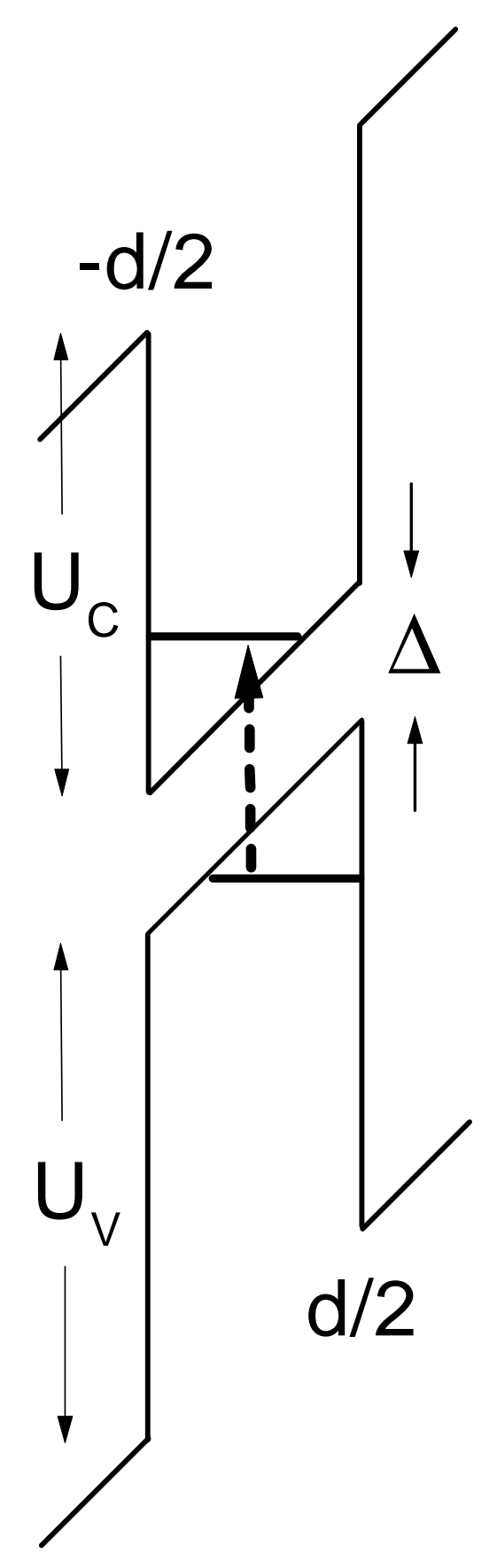

Fig.1 O.E.Raichev et al. Energy spectrum ... 


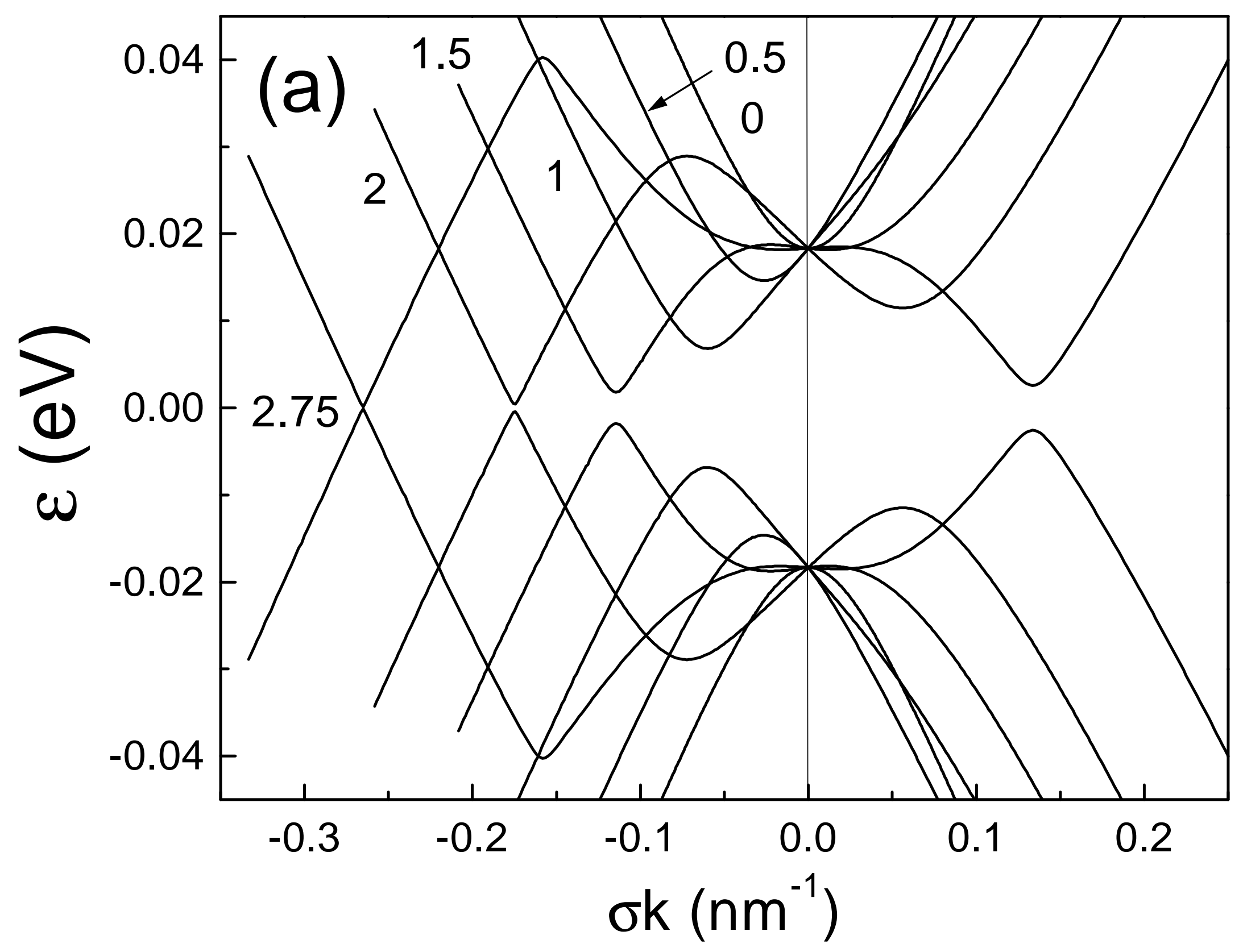

Fig.2 O.E.Raichev et al. Energy spectrum... 


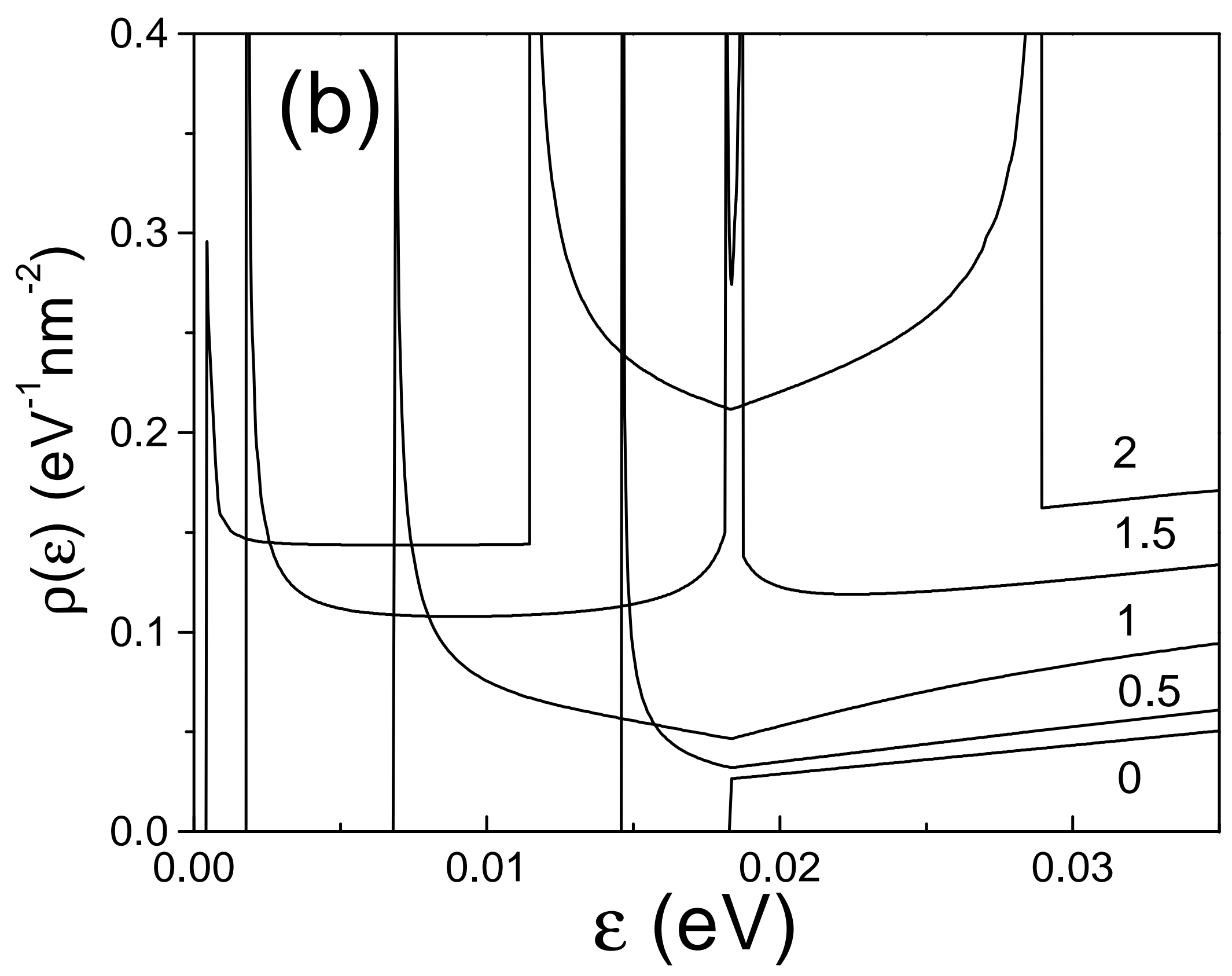

Fig.2. O.E.Raichev et al. Energy spectrum... 


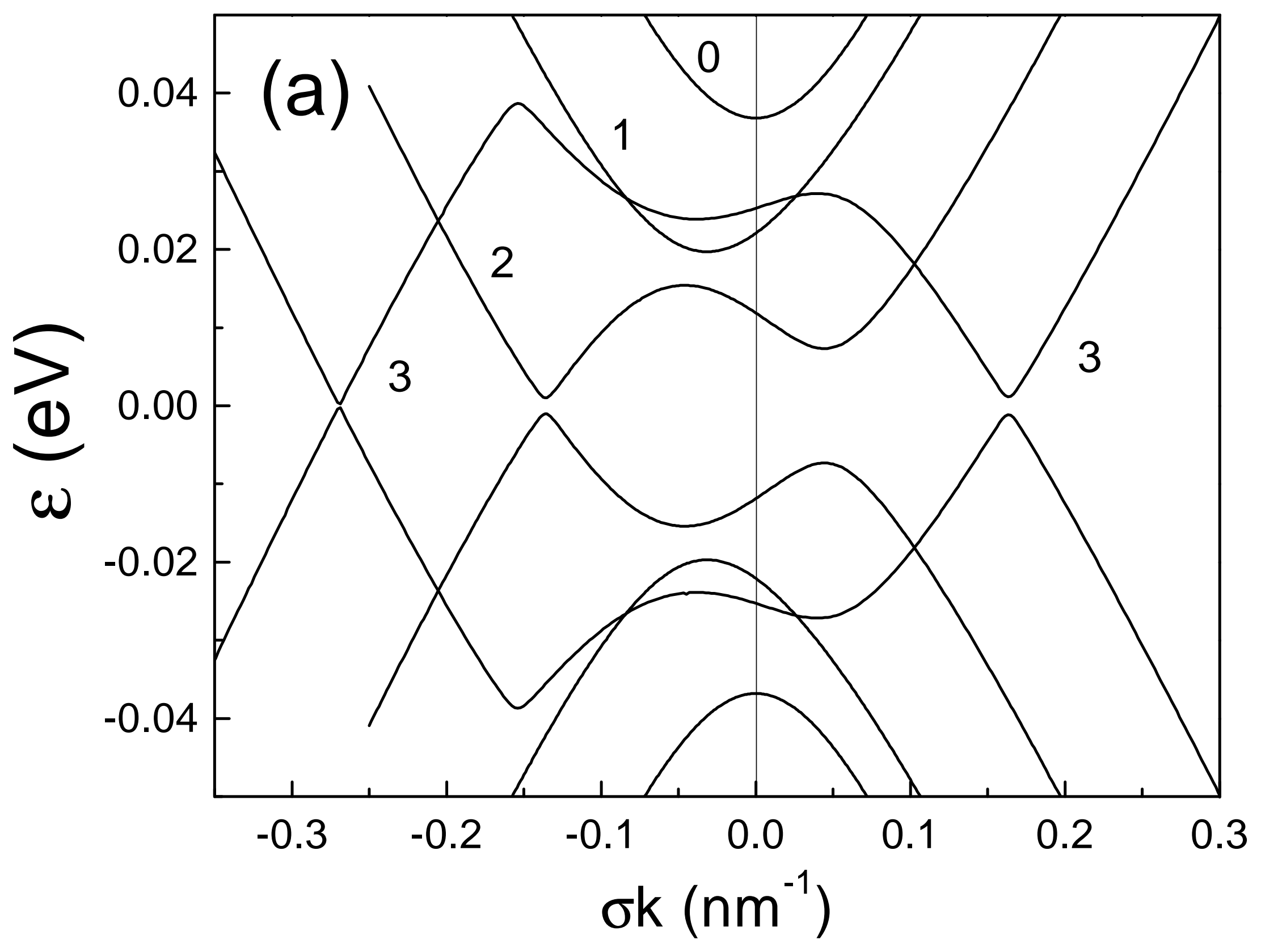

Fig.3 O.E.Raichev et al. Energy spectrum... 


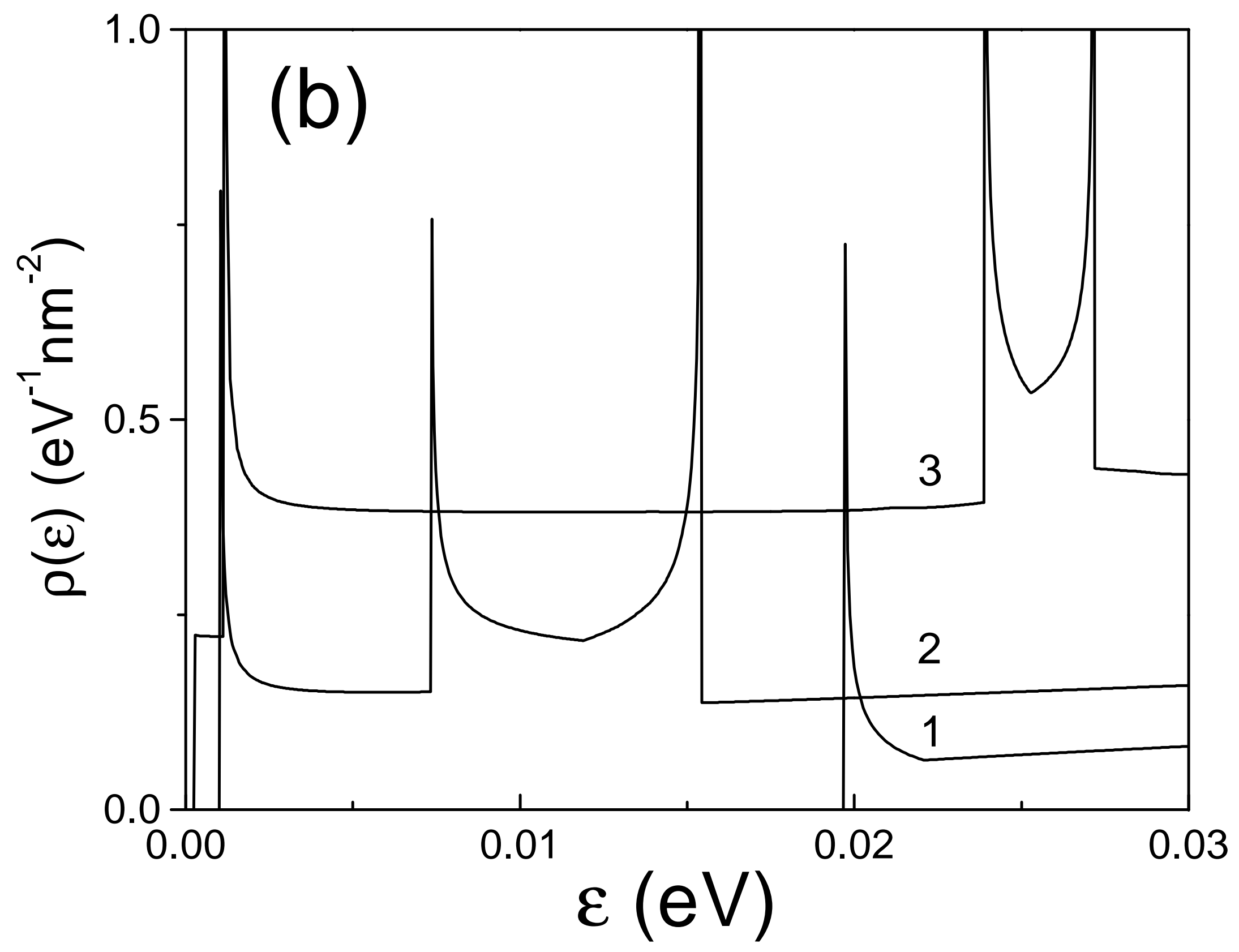

Fig.3. O.E.Raichev et al. Energy spectrum... 


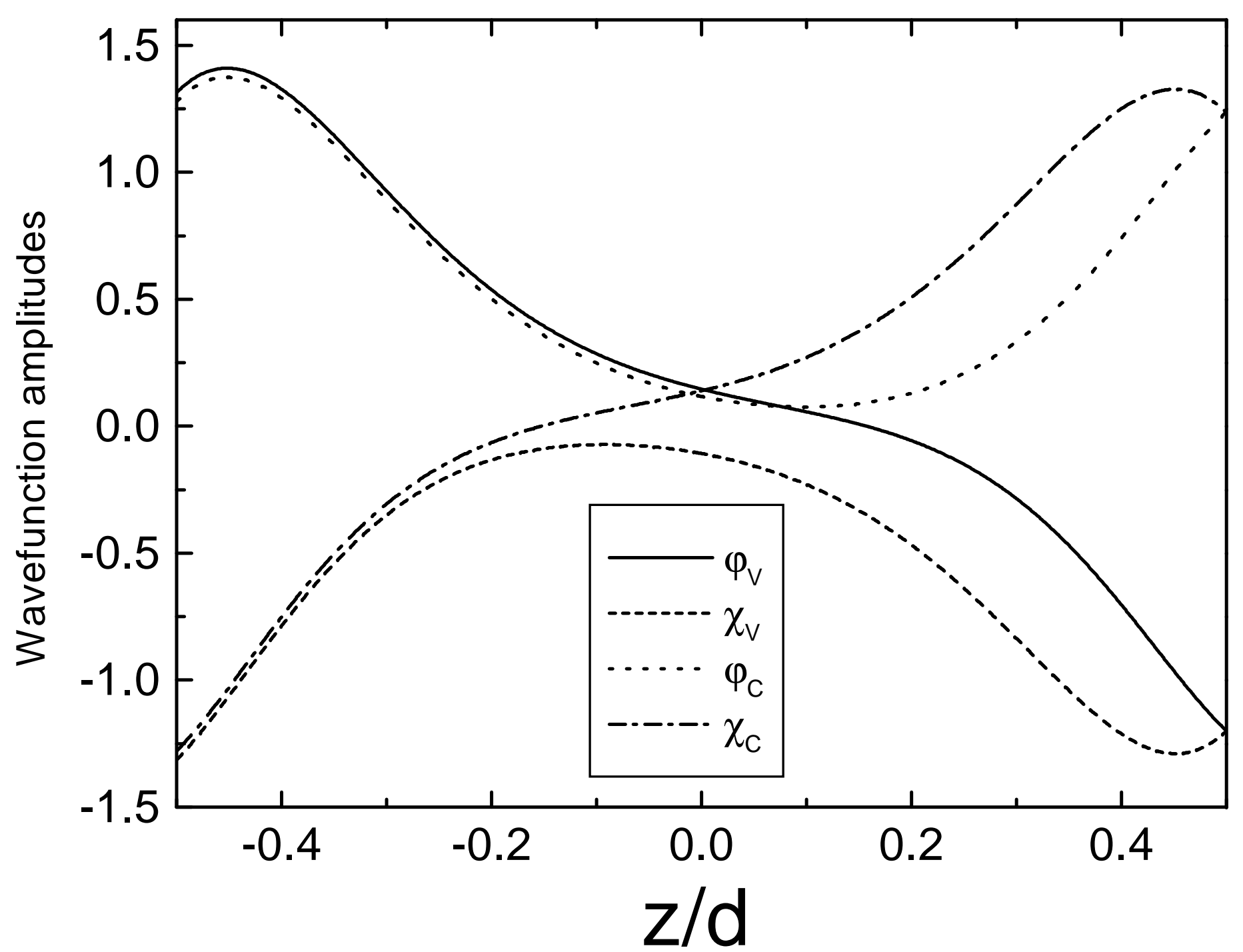

Fig.4 O.E.Raichev et al. Energy spectrum... 


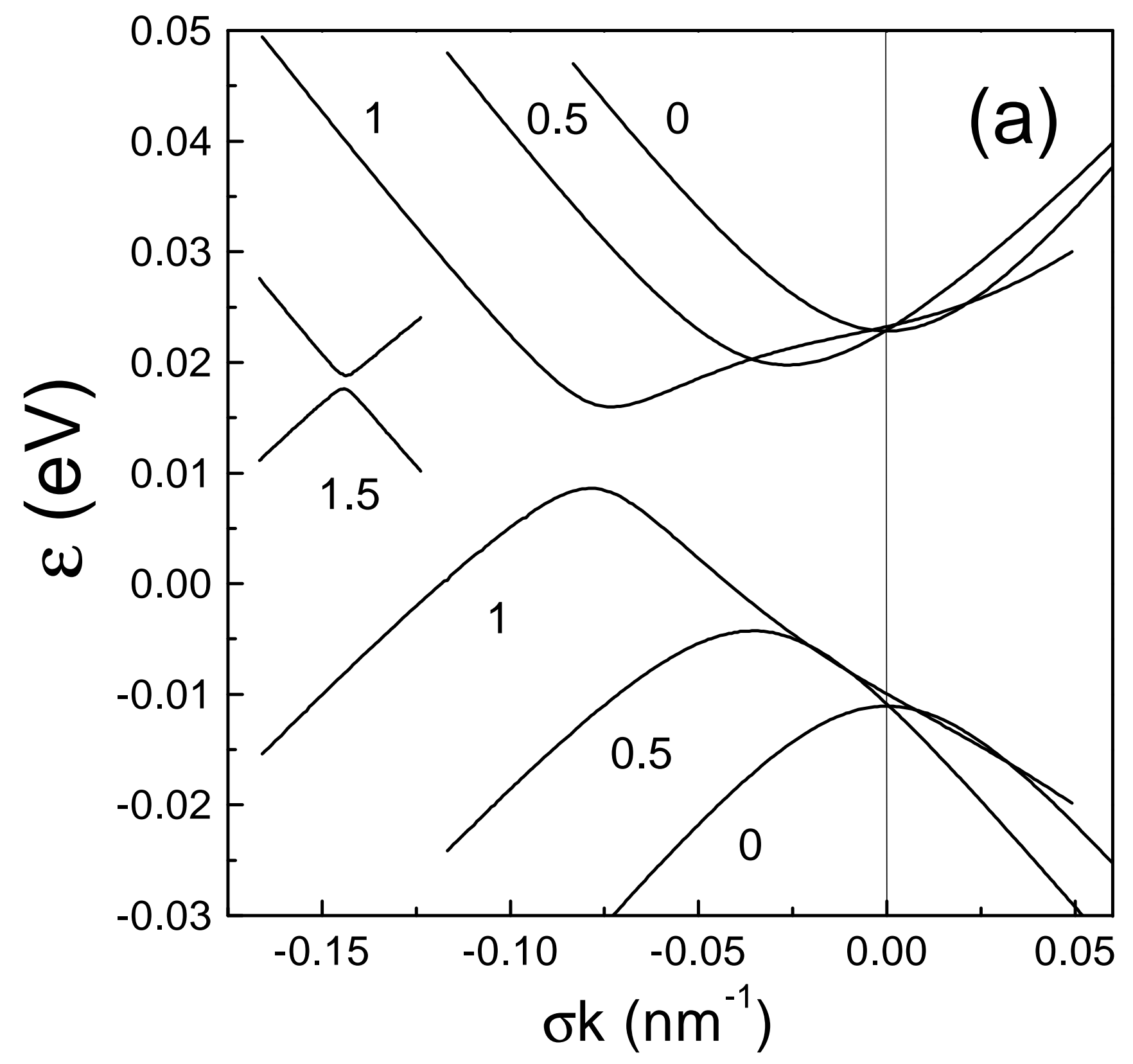

Fig.5 O.E.Raichev et al. Energy spectrum... 


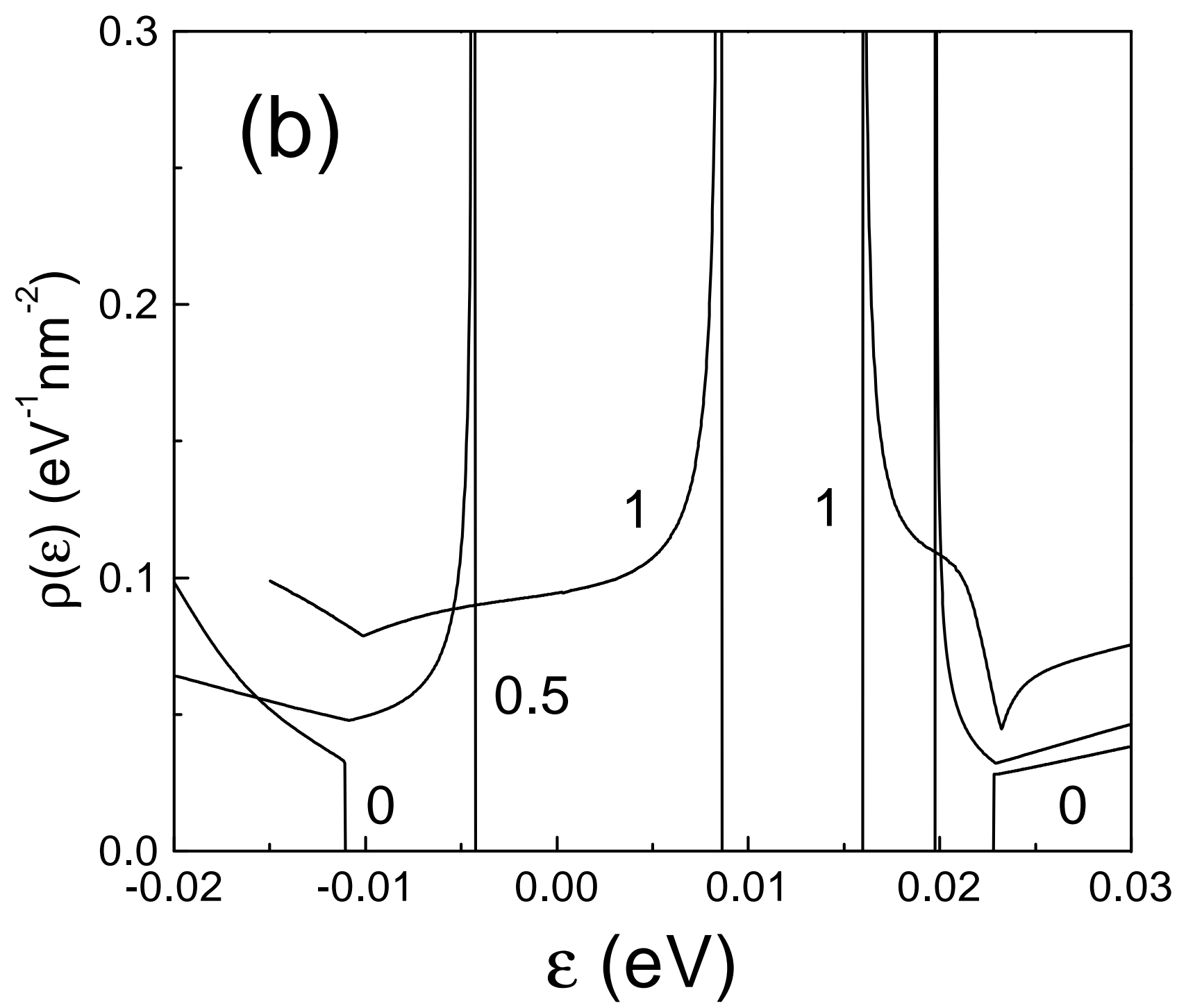

Fig.5. O.E.Raichev et al. Energy spectrum... 


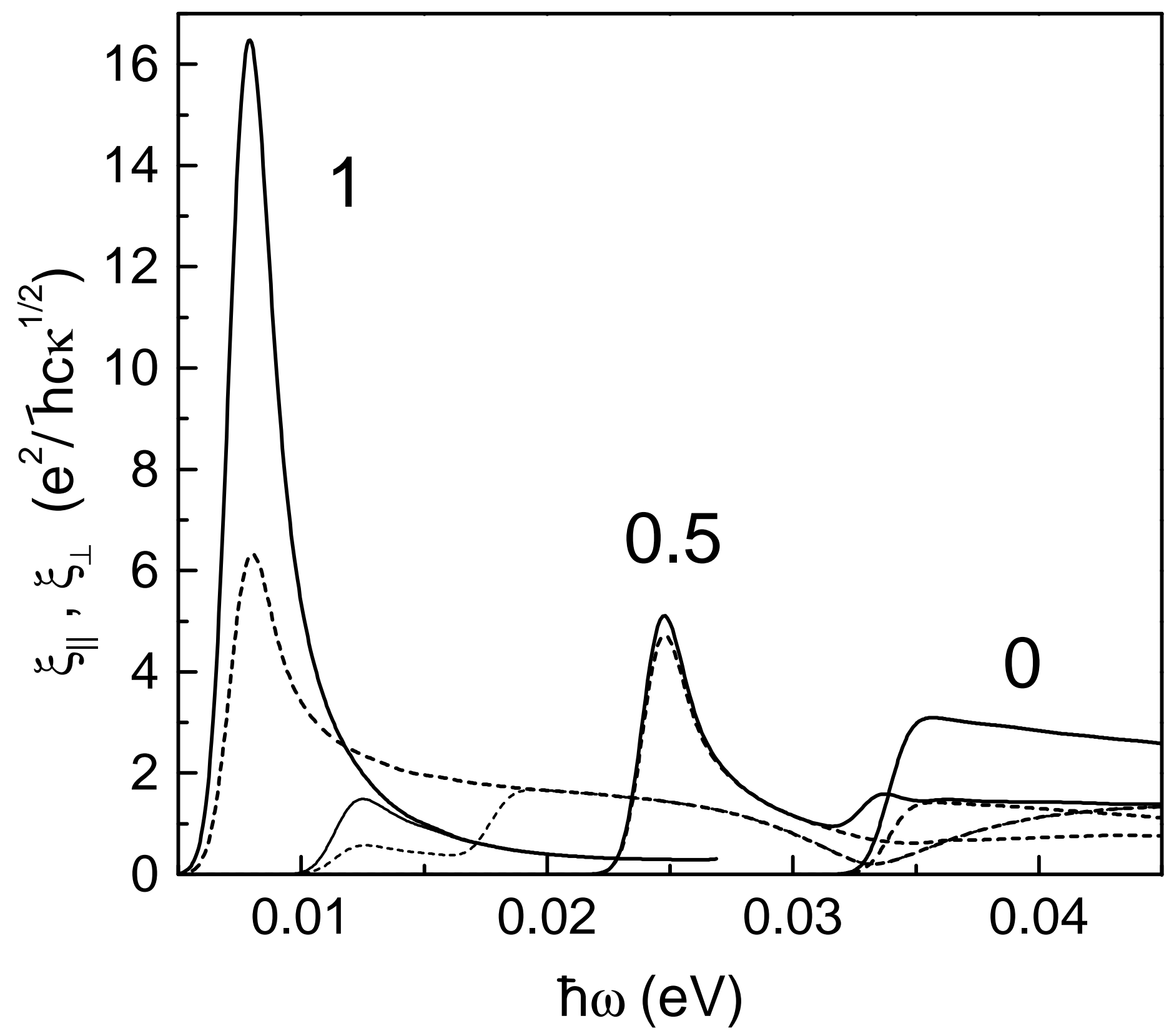

Fig.6 O.E.Raichev et al Energy spectrum ... 


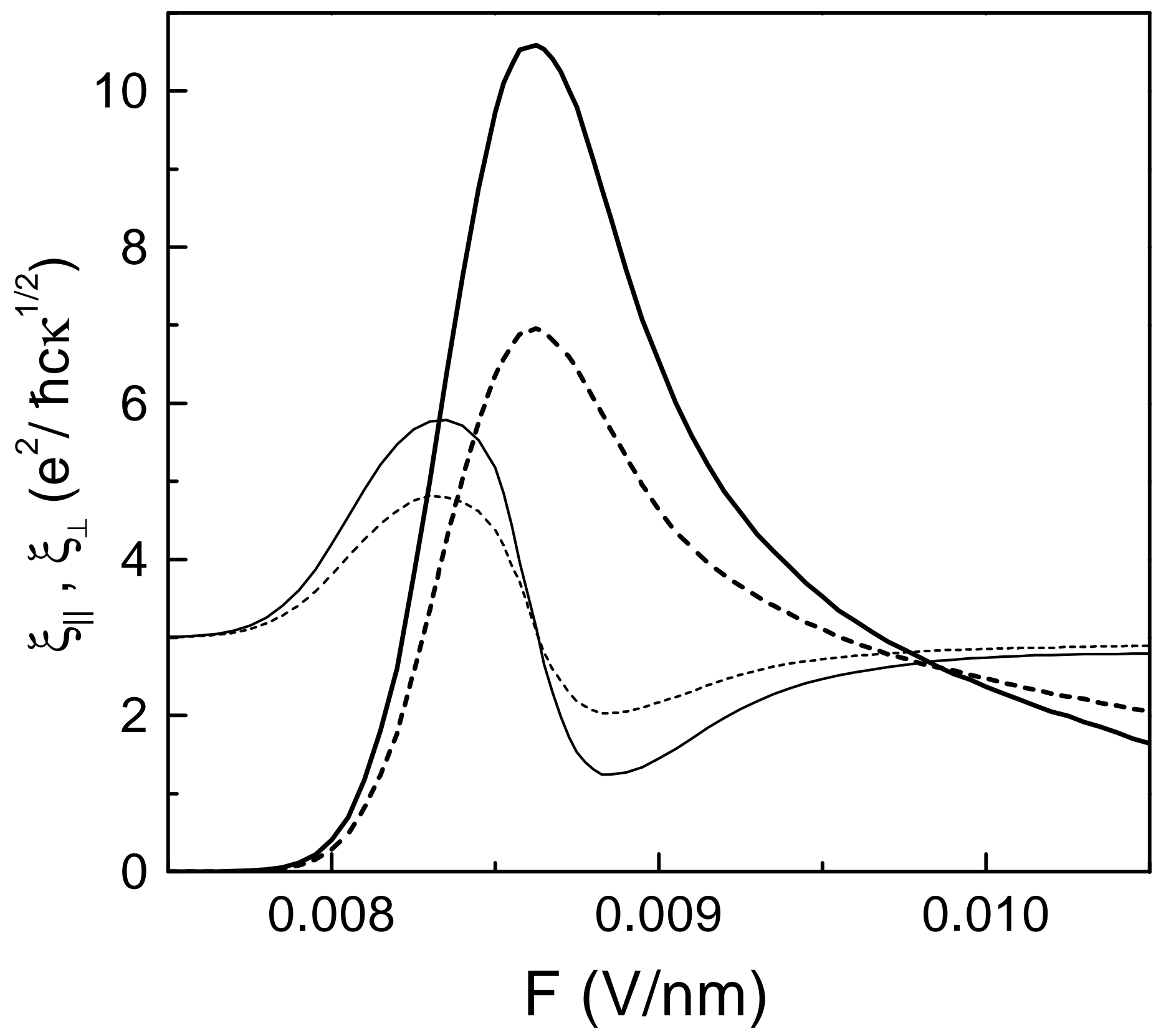

Fig.7 O.E.Raichev et al. Energy spectrum ... 